\title{
Improving Phase Measurement Procedures for Pump-Probe Experiments
}

\author{
Cara P. Perkins \\ Office of Science, Science Undergraduate Laboratory Internship Program \\ Merrimack College \\ SLAC National Accelerator Laboratory \\ Menlo Park, California
}

August 20, 2010

Prepared in partial fulfillment of the requirement of the Office of Science, Department of Energy's Science Undergraduate Laboratory Internship under the direction of Josef C. Frisch of the Linac Coherent Light Source group at SLAC National Accelerator Laboratory.

Participant:

Signature

Research Advisor:

Signature 


\section{TABLE OF CONTENTS}

Abstract.........................................................................

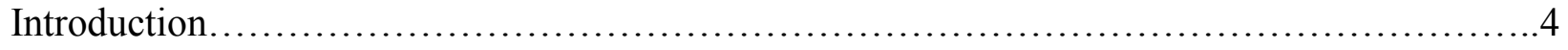

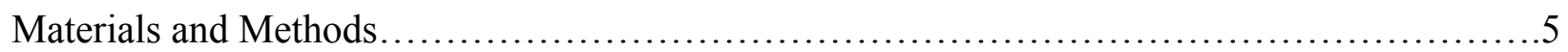

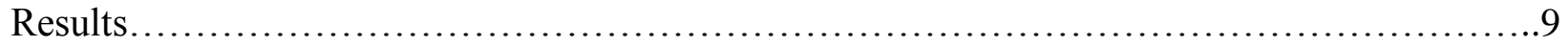

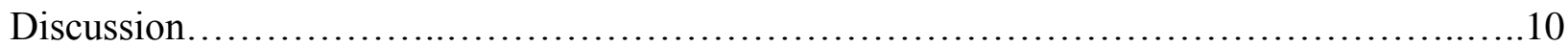

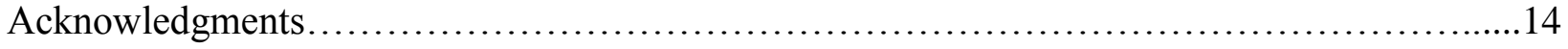

References...............................................................................

Figures and Tables.............................................................. 16 


\begin{abstract}
Improving Phase Measurement Procedures for Pump-Probe Experiments. CARA P. PERKINS (Merrimack College, North Andover, MA, 01845), JOE FRISCH (SLAC National Accelerator Laboratory, Menlo Park, CA 94025).

Pump-probe experiments use a visible laser to excite an atom or molecule, while an Xray pulse measures its shape. The phases and pulse times of each beam are used to calculate the object's positing at a given time - a moving picture of the chemical reaction. Currently, the fastest X-ray pulses can travel a time-length of five femtoseconds. However, present-day phase measurements can only be done as quickly as 50 femtoseconds. The purpose of this research is to explore ways in which phase-timing measurements can be improved. Three experiments are undergone to find the key factors in phase-timing. Different frequency mixers, the radio frequency (RF) components used for phase measurement, are tested for the highest sensitivity. These same mixers are then tested using two different power splitters for the lowest noise-tosensitivity ratio. Lastly, the temperature dependency of phase is explored by testing each component at a range of temperatures to see how the phase is affected. This research demonstrated that certain mixers were more sensitive than others; on average, one mixer performed the best with a sensitivity of $0.0230 \mathrm{~V} / \mathrm{ps}$. The results also showed that that same mixer combined with one splitter gave the best noise-to-sensitivity ratio overall with an average of $6.95 \mathrm{E}-04 \mathrm{fs} / \sqrt{ }(\mathrm{Hz})$. All the components tested exhibited a temperature-dependent phase change (ranging from 1.69 to $81.6 \mathrm{fs} /{ }^{\circ} \mathrm{C}$ ); the same mixer that performed at the highest sensitivity with the least noise had a significantly greater phase change than the other two. In conclusion, the experiments showed that a temperature-controlled environment is most appropriate for phase measurement. They also demonstrated that mixers are not significantly noisy and that certain types of mixers may perform better than others, which could be accounted for in their construction. The results of this research encourage further investigation into the study of different mixers and other RF components used in pump-probe experiments.
\end{abstract}




\section{INTRODUCTION}

The Linac Coherent Light Source (LCLS) at the Stanford Linear Accelerator Center (SLAC) National Accelerator Laboratory is the fastest, shortest pulse, highest-energy X-ray laser in the world. The LCLS shoots a beam of electrons through a specific type of magnets called undulators that create an X-ray. Initially, all the electrons begin traveling at different times. The electrons exhibit energy changes according to the effect that the electric field has on them. Those with higher energies move faster than those with lower energies. Eventually, the electrons "bunch" together at X-ray wavelengths, forming the shortest X-ray pulses yet. These fast, short X-ray pulses are used to carry out a pump-probe experiment. A pump-probe experiment triggers molecules with a visible laser and then uses the X-ray laser pulse to measure them a very short time later. Each laser beam travels through a resonance cavity that gives off a radio frequency (RF) signal containing the beam's phase information. As the beams pass through the experimental target, they trigger the detection system that measures their respective timing. By measuring the beams' phases and times relative to a reference point, the time dependence of the way the molecules move can be found, creating a movie of the chemical reaction.

This system of keeping the beams in time with respect to phase works for pulse times of about 50 femtoseconds. The LCLS can generate X-ray pulses as quickly as five femtoseconds, and it is believed that one can generate a visible beam of the same time-length [1]. These super fast lasers, however, are of no good if their phase and time measurements are not synchronized.

The purpose of this investigation is to improve the accuracy and speed with which phase is measured. A series of tests is undergone using signal generators, RF components, an oscilloscope, a voltmeter, and a spectrum analyzer. The ideal phase measurement setup has the lowest possible ratio of frequency noise to sensitivity and the least amount of phase drift. The 
sensitivity is simply a reading of the average voltage out of the system per unit of time.

Frequency noise is defined as fluctuations in a signal reading due to disturbances such as vibrations [2], while phase drift is defined as the variation in phase with time due to changes in the environment, mainly temperature.

\section{MATERIAlS ANd MethodS}

\section{Sensitivity}

The first experiment in this project tests three different frequency mixers, the components used for phase measurement in pump-probe experiments, at a variety of RF and local oscillator, LO, power levels to determine the sensitivity, or the voltage per time, of the electronic configuration using an oscilloscope. A mixer takes the two waveforms from the RF and LO inputs and outputs the intermediate frequency (IF) signal, carrying the sum and difference frequencies:

$$
\begin{aligned}
\text { Output }_{I F} & =2 \operatorname{Cos}\left(\omega_{L O} \mathrm{t}\right) \cdot \operatorname{Cos}\left(\omega_{R F} \mathrm{t}\right) \\
& =\operatorname{Cos}\left(\left(\omega_{L O}-\omega_{R F}\right) \mathrm{t}\right)+\operatorname{Cos}\left(\left(\omega_{L O}+\omega_{R F}\right) \mathrm{t}\right)
\end{aligned}
$$

Two of the mixers tested are Level 17 and the last is Level 10. The optimal power settings designated by the manufacturers for Level 17 and Level 10 mixers are $17 \mathrm{dBm}$ LO and $10 \mathrm{dBm}$ RF, and $10 \mathrm{dBm}$ LO and $5 \mathrm{dBm}$ RF, respectively [3]. For the purpose of this paper, the Mini-Circuits® Level 17 frequency mixer (model ZX05-1HW+), the Mini-Circuits ${ }^{\circledR}$ Level 17 frequency mixer (model ZFM-4H-S+), and the Mini-Circuits® Level 10 frequency mixer (model ZFM-150) will be referred to as the first, second, and third mixers, respectively. The mixers are tested at ranges of 5 to $20 \mathrm{dBm} \mathrm{LO}$ and 0 to $15 \mathrm{dBm}$ RF inputs. (The third mixer is also tested at an LO input of $0 \mathrm{dBm}$ since it is speculated to operate at lower power settings.) 
Two signal generators at slightly different frequencies provide the RF (at $476 \mathrm{MHz}$ ) and LO (at $476.01 \mathrm{MHz}$ ) power inputs to the mixer. Early measurements indicated that the two signal generators could not achieve power levels higher than $15 \mathrm{dBm}$. To obtain the higher power levels, a $20 \mathrm{dBm}$ gain RF amplifier (Mini-Circuits® ZHL-2010+) is added after each signal generator, followed by a $470 \mathrm{MHz}$ low pass filter (Mini-Circuits® VLFX-470). This filter blocks the high frequency harmonics generated by the components themselves, but is not strong enough to eliminate the $476 \mathrm{MHz}$ signal. The output of the mixer travels through a $98 \mathrm{MHz}$ low pass filter (Mini-Circuits ${ }^{\circledR}$ SLP-100+) before the oscilloscope. This low pass filter ensures that the frequency read off the oscilloscope is a reading of the difference in frequencies of the RF and LO signals only (which should be $10 \mathrm{kHz}$ in this case) (Figure 1). The signal creates a sine wave in the screen of the oscilloscope (Figure 2). The slope of the waveform taken at the zero-crossing (Volts/picoseconds) and the frequency of the incoming signal (the difference frequency) are read off the oscilloscope (Figure 3). The inverse of the difference frequency multiplied by the slope gives the number of volts per period of the waveform. Multiplying this by the frequency of the RF signal, gives a value in volts per picoseconds - the power sensitivity $(\mathrm{S})$ and the difference between the RF and LO inputs:

$$
\begin{aligned}
& \text { Volts per Period }[\mathrm{V}]=\left(1 / f_{\text {diff }}\right)\left[\mathrm{ps}^{-1}\right] \cdot \text { Slope }[\mathrm{V} / \mathrm{ps}] \\
& \boldsymbol{S}[\mathrm{V} / \mathrm{ps}]=\text { Volts per Period }[\mathrm{V}] \cdot \boldsymbol{f}_{\boldsymbol{R} F}\left[\mathrm{ps}^{-1}\right]
\end{aligned}
$$

\section{Noise}

Another electronic configuration is used to find the noise of the RF components using a spectrum analyzer (Figure 4). A single signal generator is followed by the same RF amplifier and $470 \mathrm{MHz}$ low pass filter (for the same reasons as before), and a power splitter to provide the LO and RF inputs to the mixer. A phase shifter allows the user to adjust the phase of the RF signal 
going into the mixer. Again, the mixer output travels through the $98 \mathrm{MHz}$ low pass before reaching the spectrum analyzer. Before taking measurements from the spectrum analyzer, the mixer power inputs are chosen to compare closely with those for the sensitivity measurements to calculate the noise-to-sensitivity ratios later. Also, the phase of the RF signal must be fixed at $90^{\circ}$; it is necessary to have the waveforms $90^{\circ}$ out of phase with one another because the highest phase sensitivity occurs at $90^{\circ}$. This is done by plugging the mixer output (after the $98 \mathrm{MHz}$ filter) into the voltmeter and adjusting the phase shifter until the voltage reading is $0 \mathrm{~V}$ (since the sensitivity readings were also taken at the zero-crossing).

The spectrum analyzer displays a sharply decreasing curve that levels off and becomes mostly flat (Figure 5). At any point on this plot a measurement of voltage at a given resolution bandwidth (RBW), ranging from $100 \mathrm{~Hz}$ to $100 \mathrm{kHz}$, may be taken. These measurements are used to find the noise level, NL (volts per root Hertz), of the electronic setup. Several voltage measurements taken at the same bandwidth, but different points along the curve can be used to try to find the 1/f noise, or pink noise, of the electronic setup. When referring to electronic devices, $1 / \mathrm{f}$ noise is often called pink or flicker noise and is caused by impurities in the circuitry. The more flicker noise produced using a given frequency mixer or power splitter indicates a greater amount of impurities [5].

Next, the noise-to-sensitivity ratio is calculated. Utilizing the data already gathered for the sensitivity of the mixers at given RF and LO inputs allows for the calculation of the picoseconds per root Hertz of the configuration - a noise-to-sensitivity ratio, NSR:

$$
\boldsymbol{N S R}[\mathrm{ps} / \sqrt{ }(\mathrm{Hz})]=\boldsymbol{N L}[\mathrm{V} / \sqrt{ }(\mathrm{Hz})] / \boldsymbol{S}[\mathrm{V} / \mathrm{ps}]
$$

These tests, carried out at the same LO and RF power ranges as the sensitivity tests, use the same three frequency mixers as before, and also two different splitters. The first splitter is a 
coaxial power splitter (Mini-Circuits ${ }^{\circledR}$ model ZFSC-2-1W+) which is made of transformers and other electronic devices that, presumably, have the potential to add extra noise. The second, Anaren ${ }^{\circledR}$ model (no. 40263), is made of a printed circuit (PC) board - a much simpler design.

\section{Phase Drift}

The timing system in the LCLS can easily change phase conditions; phase drift may be caused by instable temperature. For this reason, it is necessary to check for phase temperature dependencies of the RF components. This part of the project uses the same electronic setup as the noise experiment, but with one of the RF components on a temperature regulated plate (Figure 6).

At this point in the project, the sensitivity and noise measurements provide a decent understanding of what the optimal RF and LO power settings are for each mixer. This part of the experiment uses these optimal settings so that only the temperature is varying. Starting at the highest temperature reached by the temperature regulation machine, the signal is sent to the voltmeter. (The temperature regulation machine has a range of 10 to $35^{\circ} \mathrm{C}$. According to the manufacturers, all the RF components used in this experiment should operate properly in this range.) As with noise measurements, the phase is fixed at $90^{\circ}$ at this high temperature. As the temperature-controlled RF component cools, the voltage reading on the voltmeter is noted at each degree Celsius. This process continues until the temperature has reached the minimum. Then, the system is warmed up to its original temperature while the voltage reading is periodically recorded. If the phase drift of the component is temperature-dependent, the voltage reading should move away from zero as the component cools and then return to zero as it heats up to its starting temperature. This experiment is carried out for a total of eight times; the two splitters, three mixers, amplifier, and phase shifter each have a turn on the temperature-regulated 
plate. The eighth experiment tests a $10 \mathrm{ft}$. cable coil between the phase shifter and the RF input to the mixer.

Each reading off the voltmeter provides a change in voltage per degree Celsius. Taking the average change in voltage per degree Celsius and dividing it by the sensitivity gives the change in phase time per degree Celsius, or the phase drift:

$$
\text { Phase Drift }\left[\mathrm{fs} /{ }^{\circ} \mathrm{C}\right]=\left(\Delta V /{ }^{\circ} \mathrm{C}\right)_{\text {avg }} / S[\mathrm{~V} / \mathrm{fs}]
$$

\section{RESULTS}

\section{Sensitivity}

The sensitivity experiment gave 20 sensitivity measurements, corresponding with different RF and LO inputs, for each mixer. The best sensitivity measurement, $0.05620 \mathrm{~V} / \mathrm{ps}$, was taken using the first mixer at $20 \mathrm{dBm}$ LO input and $15 \mathrm{dBm}$ RF input, while the worst sensitivity reading, $0.00421 \mathrm{~V} / \mathrm{ps}$, was taken using the third mixer at $17 \mathrm{dBm} \mathrm{LO}$ and $0 \mathrm{dBm} \mathrm{RF}$. On average, the first mixer was the most sensitive at about $0.02300 \mathrm{~V} / \mathrm{ps}$, followed by the second at $0.01860 \mathrm{~V} / \mathrm{ps}$, then the third at $0.00596 \mathrm{~V} / \mathrm{ps}$ (Table 1).

\section{Noise}

Six sets of data resulted from the noise experiment. Each of the three mixers was tested for noise using each of the two splitters. Using one splitter versus another did not seem to have a great effect on the noise. While taking the data, it became clear that the plot shown on the spectrum analyzer was not actually displaying flicker noise. Although the plot looked like a 1/f plot (Figure 5), it was determined that the steep decline in noise voltage was simply a result of the averaging of lower-frequency harmonics, not eliminated by the low pass filters. This discovery led to the decision to neglect searching for the $1 / \mathrm{f}$ plot and deal specifically with the 
noise-to-sensitivity ratio. The readings off the spectrum analyzer were taken from the regions of the plot that were mostly level. Taking the average ratios for each combination showed that the first mixer combined with the first splitter had the least amount of noise with a noise-tosensitivity ratio of $6.95 \mathrm{E}-04 \mathrm{fs} / \sqrt{ }(\mathrm{Hz})$, followed by the second mixer with the second splitter at 7.47E-04 fs $/ \sqrt{ }(\mathrm{Hz})$, the first mixer with the second splitter with a ratio of $7.56 \mathrm{E}-04 \mathrm{fs} / \sqrt{ }(\mathrm{Hz})$, the second mixer with the first splitter at 9.00E-04 fs $/ \sqrt{ }(\mathrm{Hz})$, the third mixer with the first splitter at $1.62 \mathrm{E}-03 \mathrm{fs} / \sqrt{ }(\mathrm{Hz})$, and finally the third mixer with the second splitter at $1.70 \mathrm{E}-03 \mathrm{fs} / \sqrt{ }(\mathrm{Hz})$, (Table 2). For the purpose of further investigation, a second RF amplifier, followed by enough attenuation so that no power was added to the system, was inserted before the RF input of the mixer to see if the noise increased at all. The noise floor and, thus, the noise-to-sensitivity ratio did not increase with the additional amplifier.

\section{Phase Drift}

The phase drift experiment provided eight phase drift measurements - one for each of the items tested on the temperature-regulated plate. The RF component with the least amount of phase drift, $1.86 \mathrm{fs} /{ }^{\circ} \mathrm{C}$, was the second mixer, while the $10 \mathrm{ft}$. cable coil had the greatest amount of phase drift at $81.6 \mathrm{fs} /{ }^{\circ} \mathrm{C}$. Falling between these two were the first splitter at $3.78 \mathrm{fs} /{ }^{\circ} \mathrm{C}$, the third mixer at $6.95 \mathrm{fs} /{ }^{\circ} \mathrm{C}$, the amplifier at $7.88 \mathrm{fs} /{ }^{\circ} \mathrm{C}$, the second splitter at $8.5 \mathrm{fs} /{ }^{\circ} \mathrm{C}$, the phase shifter at $24.6 \mathrm{fs} /{ }^{\circ} \mathrm{C}$, and the first mixer at $26.8 \mathrm{fs} /{ }^{\circ} \mathrm{C}$, respectively (Table 3).

\section{DISCUSSION}

While reviewing the results of this project, certain things must be kept in mind. One is the fact that these experiments were done using only three mixers, while there are millions of different mixers in existence. The same is true for splitters. Also, the manufacturers of the RF 
components used in this project only provide user specifications for general applications.

Therefore, these results cannot be compared to other results since these components were not intended to be used with the fastest X-ray laser beam in the world.

\section{Sensitivity}

The sensitivity graphs show that the output voltage of all three mixers increased with the RF power (Figures 7a-f). When looking at the graphs of the sensitivity as a function of the LO power (Figures $7 b, d, f)$, one sees that once the mixers reach a certain LO power input, their outputs level off. In the case of all three mixers, the highest sensitivity occurred at the highest LO and RF power inputs tested, $20 \mathrm{dBm}$ LO and $15 \mathrm{dBm}$ RF. If a user is concerned solely with getting a maximum voltage out of a mixer, then these power levels are beneficial. However, the flat areas on the sensitivity versus LO power plots (Figures 7b, d, f) indicate regions where the power does not affect the overall sensitivity. Therefore, these settings may be better-suited for someone who is not using the mixers at very specific power levels. The results also showed that the Level 10 mixer was less sensitive than the Level 17 mixers by and order of 10 . Therefore, it may be possible that all Level 17 mixers are more sensitive than Level 10 mixers.

\section{Noise}

To better understand why these flat-lined plots are more significant, consider the noise graphs (Figures 8a-f). For example, the graphs of the first mixer noise levels show that a $10 \mathrm{dBm}$ LO input consistently gives the lowest noise-to-sensitivity ratio, and this noise level does not change much between a 5 to $10 \mathrm{dBm}$ RF input. Because the pump-probe experiment is so complicated, one cannot be very concerned with exact power levels while measuring phase. Therefore, having a range of power inputs where the noise-to-sensitivity measurement does not change significantly is very helpful. With respect to the second mixer, the lowest noise ratio is at 
$17 \mathrm{dBm} \mathrm{LO}$ with an RF range of 5 to $10 \mathrm{dBm}$. The third mixer gives the lowest noise-tosensitivity ratio at $10 \mathrm{dBm} \mathrm{LO}$ and an $\mathrm{RF}$ range of 5 to $10 \mathrm{dBm}$. Two main points of interest are within these results. One is that this flat-line plot is most visible at the $100 \mathrm{~Hz}$ RBW for the Level 17 mixers, and at the $10 \mathrm{kHz}$ RBW for the Level 10 mixer. It is also interesting that both the first and third mixers operate best at $10 \mathrm{dBm} \mathrm{LO}$ and 5 to $10 \mathrm{dBm} \mathrm{RF}$, even though the former is a Level 17 mixer and the latter is a Level 10 mixer. This could have resulted from the way the mixers were built, with respect to both their mechanical blueprints and their materials information that Mini-Circuits ${ }^{\circledR}$ has the right to withhold from customers (and does withhold from customers).

\section{Phase Drift}

The results of the phase drift experiment show that all the RF components tested have at least some temperature dependency, some greater than others (Table 3). These results imply that the easiest way to avoid phase drift in the LCLS system is to regulate temperature, eliminating most, if not all, of the phase drift in the RF components. Because the cable coil showed the highest phase drift as a function of temperature, limiting the amount of cable used would also help reduce phase drift. (There are other types of cables in existence that claim to have lower

noise levels, but they have yet to be tested.) Why some components have more phase drift with temperature may have to do with the materials they are made of or how they are built. For example, the phase drift of the first mixer is greater than that of the other two mixers by an order of magnitude, even though this same mixer performed best during the sensitivity and noise experiments. Again, Mini-Circuits ${ }^{\circledR}$ does not reveal details of the construction of its components so there is no way to say one construction or one material is better than another. 


\section{Conclusion}

This project demonstrates one way to test frequency mixers, but it does not provide an answer for what type of mixer would be the "best"- especially considering how differently these mixers performed. There are too many unknowns with respect to the construction of frequency mixers to say that one design is better than another. Further research on this subject may provide a better understanding of the performance of mixers that would eliminate the need to test individual mixers for noise-to-sensitivity ratios.

But what do all these results mean for pump-probe experiments at the LCLS?

Multiplying the square-root of the $200 \mathrm{kHz}$ RBW, used by the LCLS system, by the noise-tosensitivity results shows that the noise produced by the mixers affects the phase-time measurements by less than one femtosecond (Table 2). Therefore, neither mixers nor amplifiers (as noted earlier) are main contributors to noise, and will not significantly disrupt the RF signals of the pump and probe beams; other components have yet to be tested. Lastly, the phase drift measurements are on the femtosecond scale (Table 3). By maintaining a temperature within onetenth of a degree Celsius, one can not only harness the phase drift of the system, but also keep the phase timing down at the LCLS's five-femtosecond scale. 


\section{ACKNOWLEDGEMENTS}

I would like to express my greatest appreciation to the people responsible for the overall production and success of this project, completed at SLAC National Accelerator Laboratory during the months June through August, 2010. Thank you to the U.S. Department of Energy for funding the Science Undergraduate Laboratory Internships (SULI), and thank you to SLAC for organizing this SULI program. I would also like to thank Stanford University for its contribution to the program, including the provision of housing. I must also acknowledge my laboratory partner and fellow intern, Michael Whalen, and the laboratory technician, George Burgueno, for their assistance with this project. Lastly, a special thank you goes to Joe Frisch, the creator of this project and my mentor. 


\section{REFERENCES}

[1] A. Brachmann, C. Bostedt, J. Bozek, R. Coffee, F.J. Decker, Y. Ding, D. Dowell, P. Emma, J. Frisch, S. Gilevich, G. Haller, G. Hays, Ph. Hering, B. Hill, Z. Huang, R. Iverson, E. Kanter, B. Kraessig, H. Loos, A. Miahnahri, H.-D. Nuhn, A. Perazzo, M. Petree, D. Ratner, R. Santra, T. Smith, S. Southworth, J. Turner, J. Welch, W. White, J. Wu, L. Young: SLAC National Laboratory, Stanford CA, USA, J. M. Byrd, G. Huang, R. Wilcox: LBNL, Berkley CA, USA, "Femtosecond Operation of the LCLS for User Experiments," accelconf.web.cern.ch/accelconf/IPAC10/papers/tupe066.pdf.

[2] 'Noise (Electronics)," August 5, 2010, http://en.wikipedia.org/wiki/Noise_\%28electronics\%29.

[3] "Frequently asked questions about phase detectors (AN-41-001)," Mini-Circuits @, July 17, 2010, https://www.minicircuits.com/pages/pdfs/an41001.pdf.

[4] F. Marci, C. Marci, "Mixer Basics Primer," http://webcache.googleusercontent.com/search?q=cache:VL2K6UXjUUJ:www.markimicrowave.com/menus/appnotes/mixer_basics_primer.pdf+purpose+of $+\mathrm{a}+$ microwave+mixer\&hl=en\&gl=us.

[5] “Flicker Noise,” July 10, 2010, http://en.wikipedia.org/wiki/Flicker_noise. 


\section{TABLES AND FIGURES}

Average Sensitivity of the Mixers

\begin{tabular}{|c|c|}
\hline Mixer Number & Sensitivity (V/ps) \\
\hline 1 & 0.02300 \\
\hline 2 & 0.01860 \\
\hline 3 & 0.00596 \\
\hline
\end{tabular}

Table 1. This table displays the average power sensitivity of each of the three mixers.

\section{Average Noise of Mixer and Splitter Combinations}

\begin{tabular}{|l|c|c|}
\hline Combination & Noise-to-Sensitivity Ratio (fs $/ \sqrt{ }(\mathrm{Hz}))$ & LCLS Timescale at 200 kHz RBW (fs) \\
\hline \#1 Mixer, \#1 Splitter & $6.95 \mathrm{E}-04$ & 0.311 \\
\hline \#1 Mixer, \#2 Splitter & $7.56 \mathrm{E}-04$ & 0.338 \\
\hline \#2 Mixer, \#1 Splitter & $9.00 \mathrm{E}-04$ & 0.402 \\
\hline \#2 Mixer, \#2 Splitter & $7.47 \mathrm{E}-04$ & 0.334 \\
\hline \#3 Mixer, \#1 Splitter & $1.62 \mathrm{E}-03$ & 0.724 \\
\hline \#3 Mixer, \#2 Splitter & $1.70 \mathrm{E}-03$ & 0.760 \\
\hline
\end{tabular}

Table 2 . Here are the average noise-to-sensitivity ratios of each mixer / splitter combination.

\section{Average Phase Drift of Different Components}

\begin{tabular}{|c|c|}
\hline RF Component & Phase Drift $\left(\mathrm{fs} /{ }^{\circ} \mathrm{C}\right)$ \\
\hline \#1 Mixer & 26.8 \\
\hline \#2 Mixer & 1.86 \\
\hline \#3 Mixer & 6.95 \\
\hline \#1 Splitter & 3.78 \\
\hline \#2 Splitter & 8.50 \\
\hline RF Amplifier & 7.88 \\
\hline Phase Shifter & 24.6 \\
\hline 10 ft. Cable Coil & 81.6 \\
\hline
\end{tabular}

Table 3. Table 3 shows the average change in phase time per degree Celsius for each RF component tested on the temperature regulation plate. 


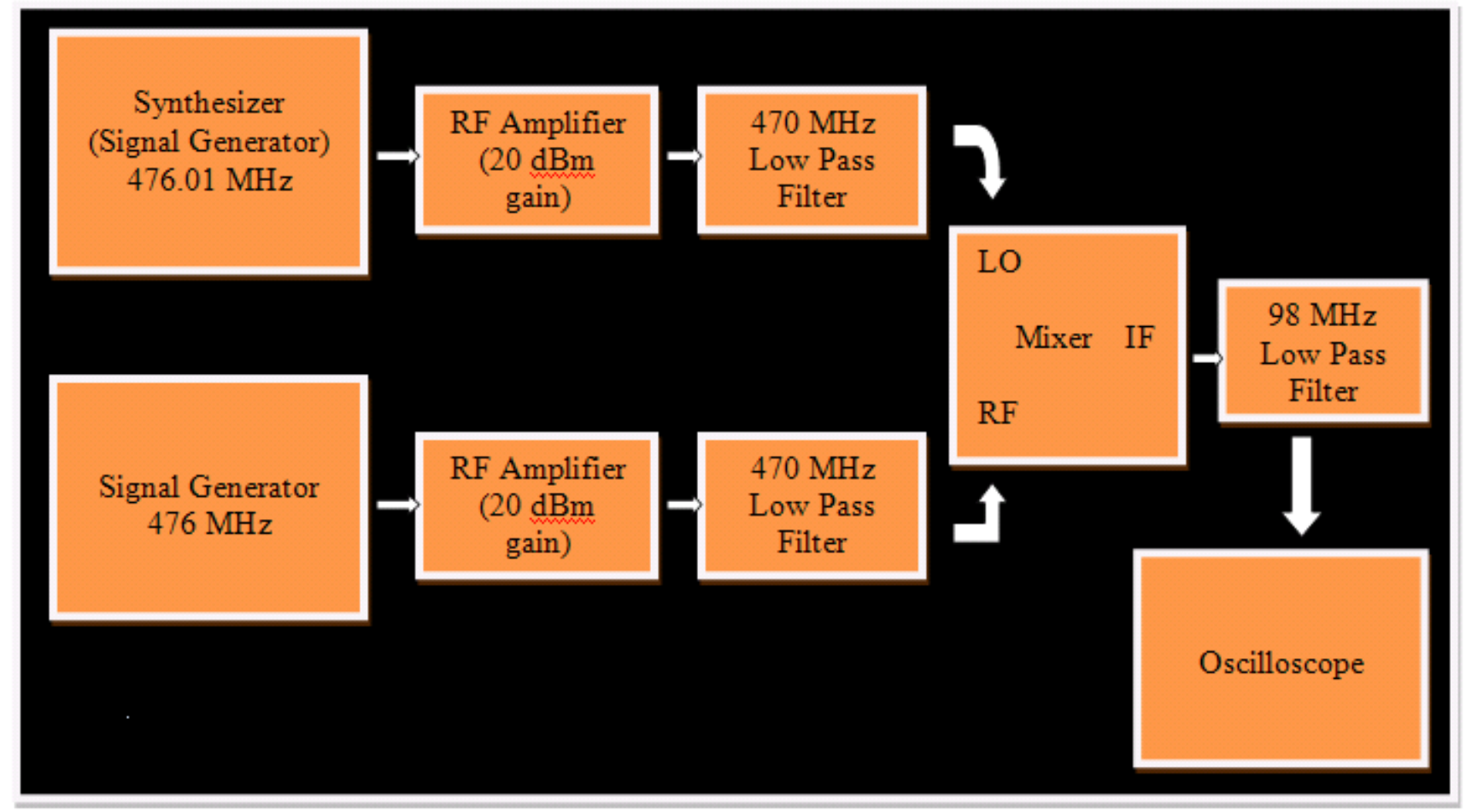

Figure 1. This is the electronic setup used to collect the power sensitivity data for each of the three frequency mixers. 


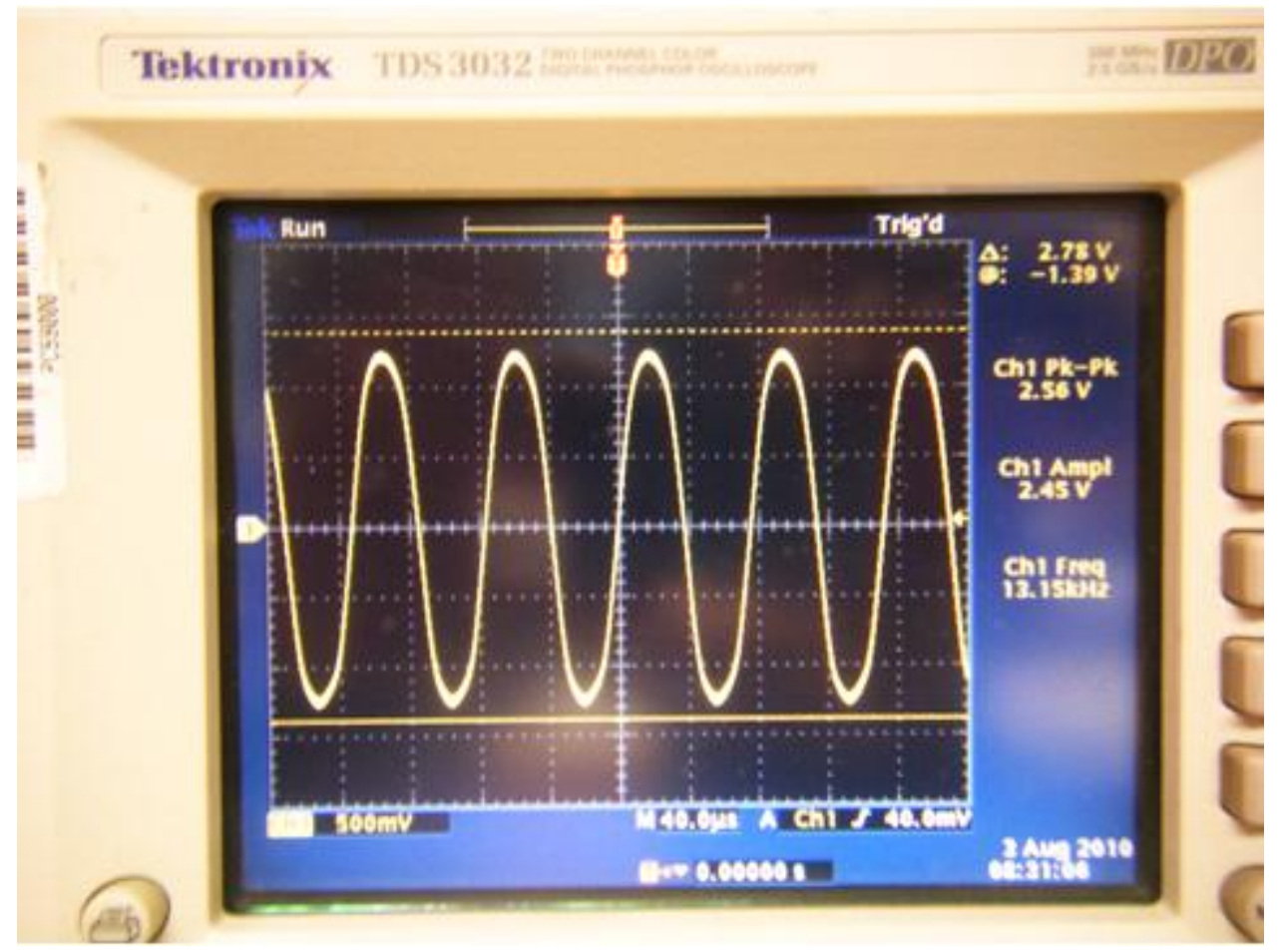

Figure 2. The signal from the IF of the mixer produces a sine waveform at the difference frequency on the oscilloscope.

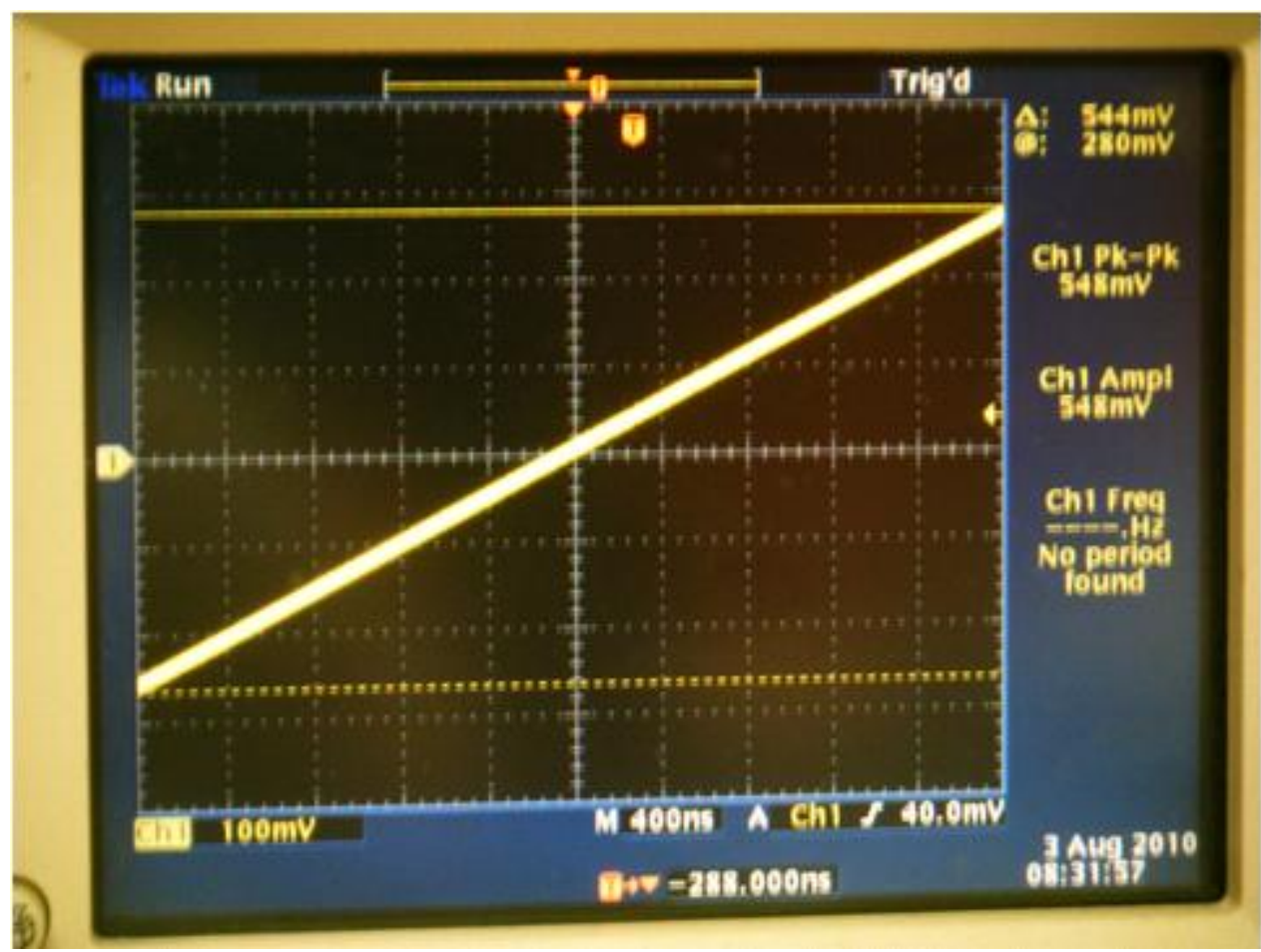

Figure 3. Zooming in at the zero-crossing gives a line with a slope of voltage per time. 


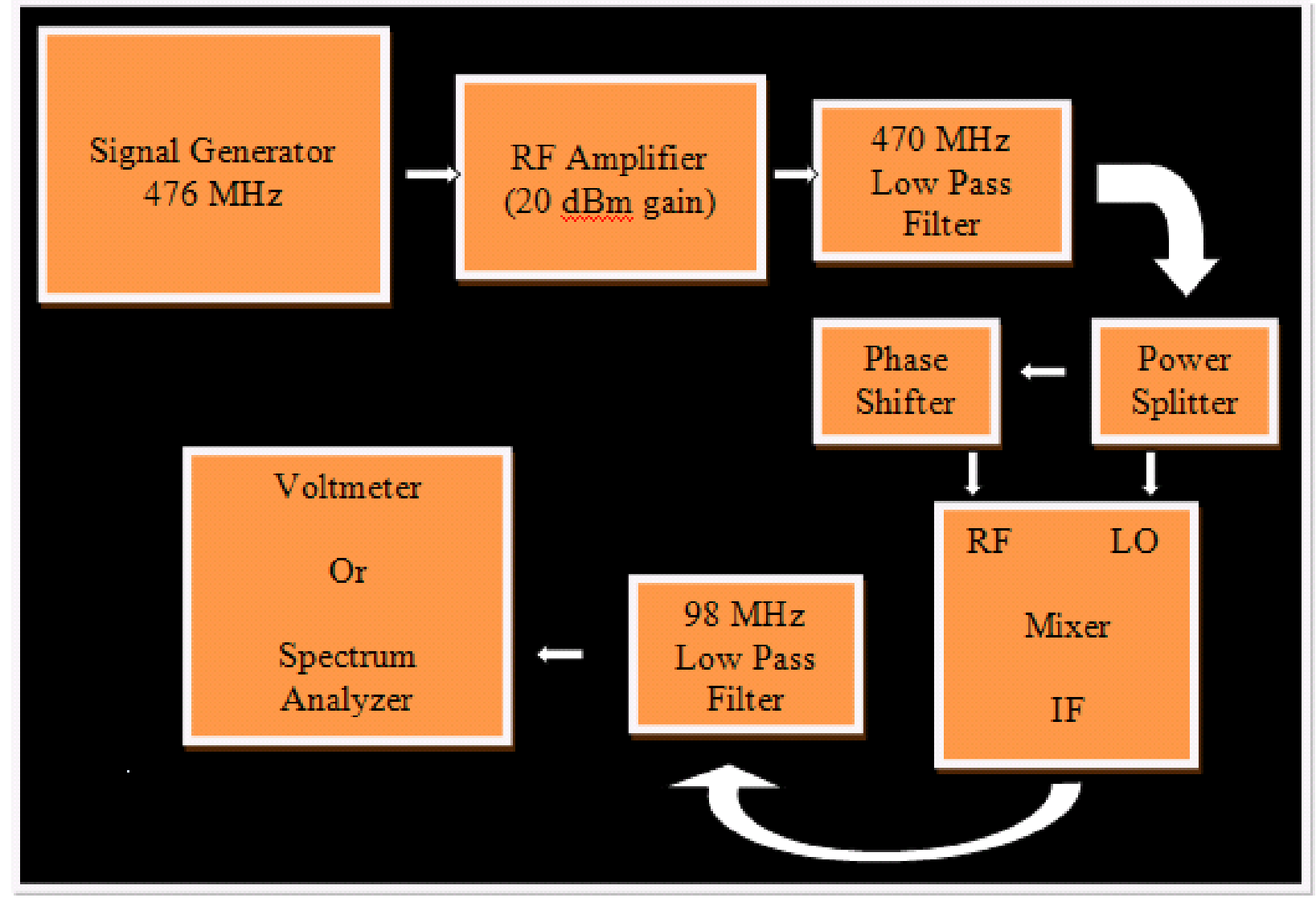

Figure 4. This is the setups used to measure the noise levels of the three mixers and two splitters. 


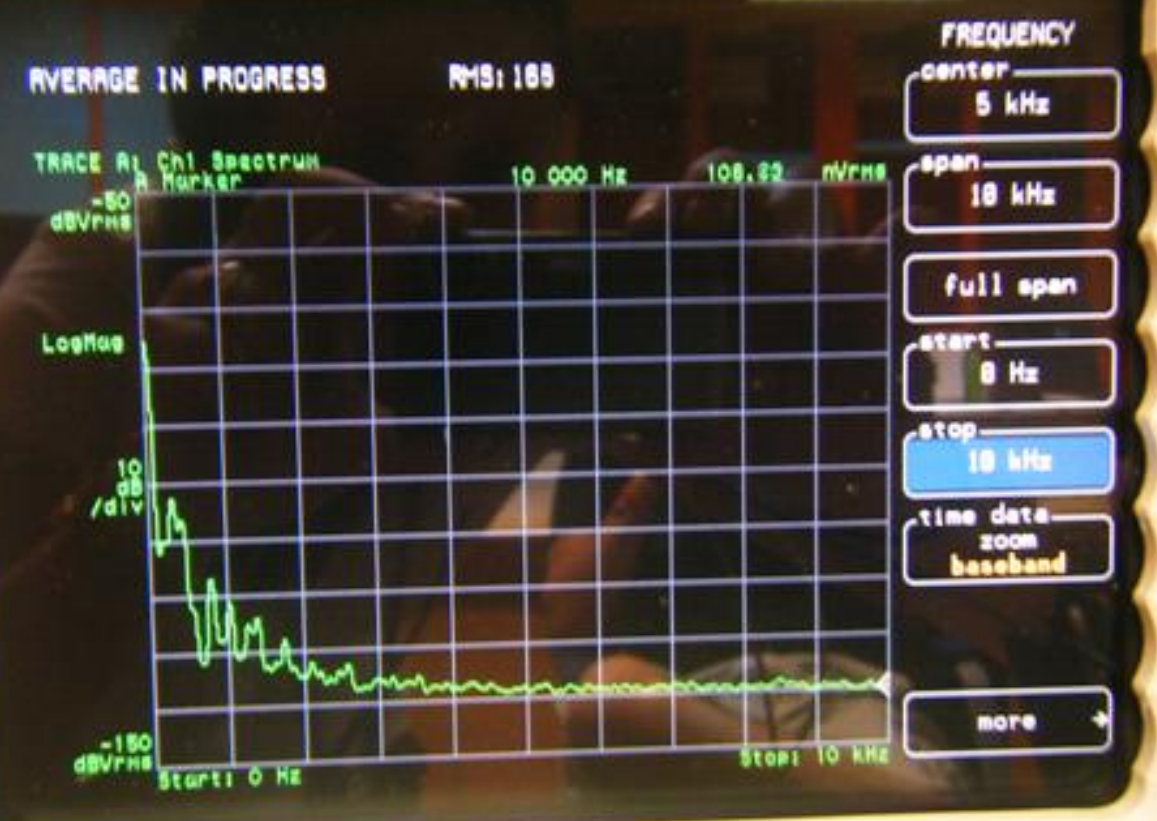

Figure 5. The spectrum analyzer displays a noise plot that resembles a 1/f plot.

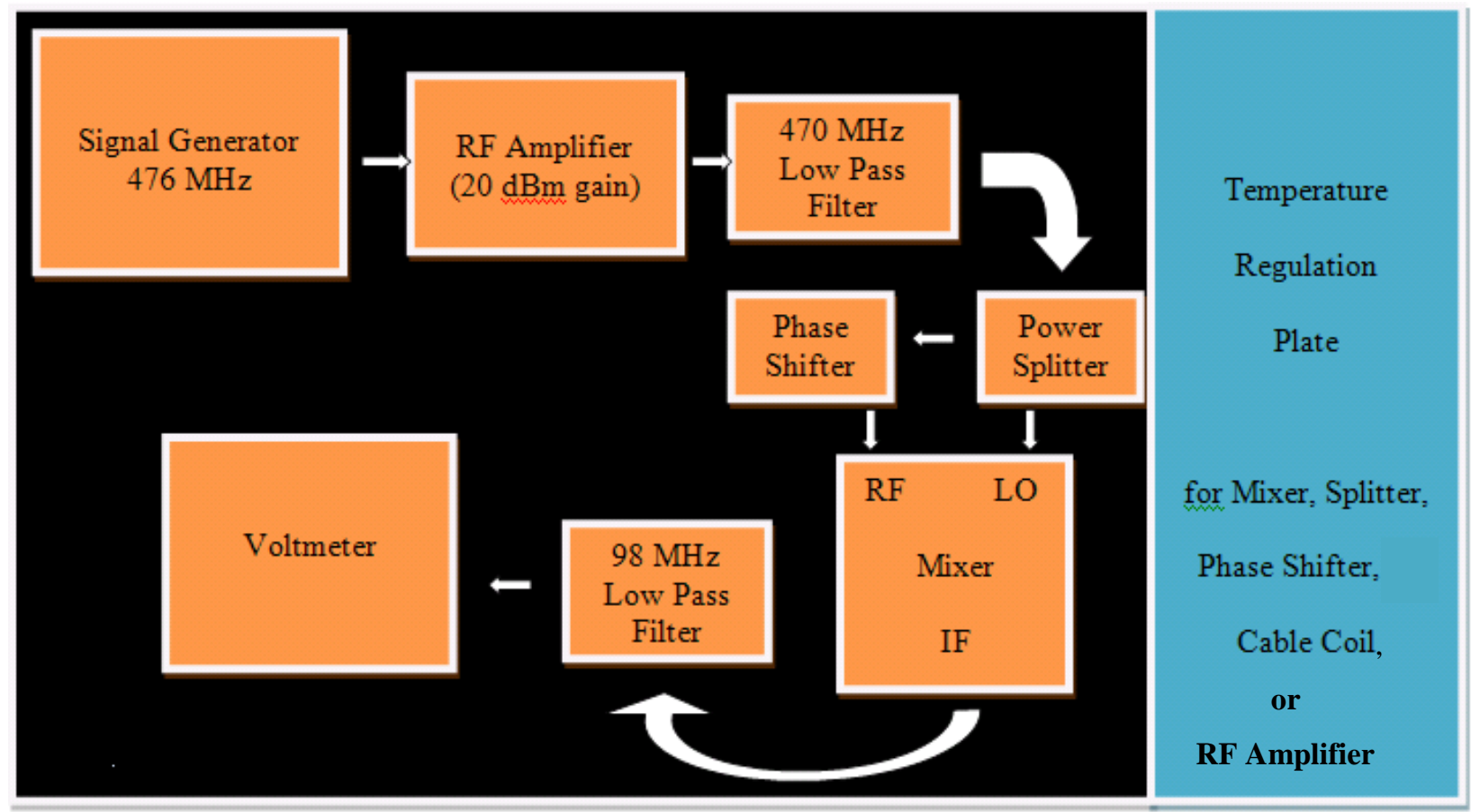

Figure 6. The phase drift setup is very similar to that of the noise experiment. Here, each of the eight components tested would take a turn on the temperature regulation plate, as shown. 


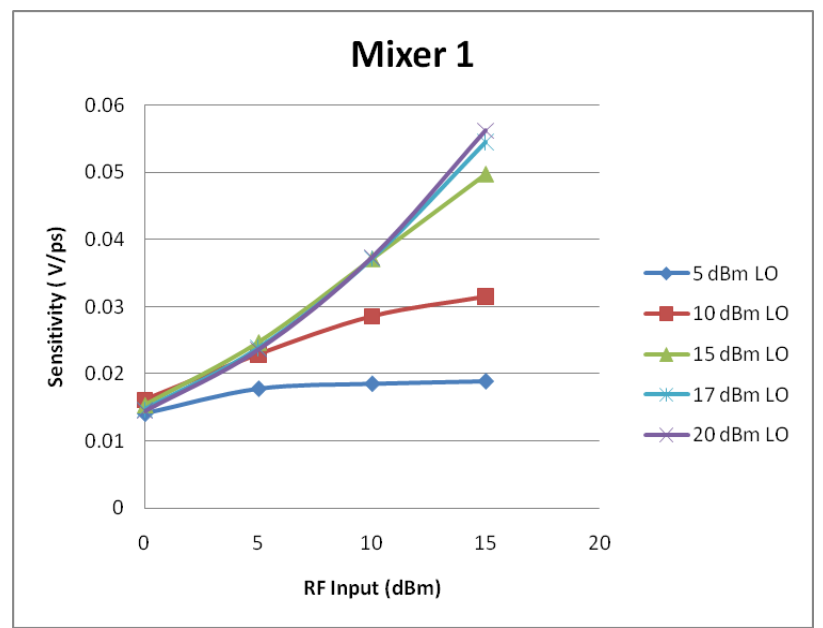

Figure 7a. Mixer 1's sensitivity vs. RF plot.

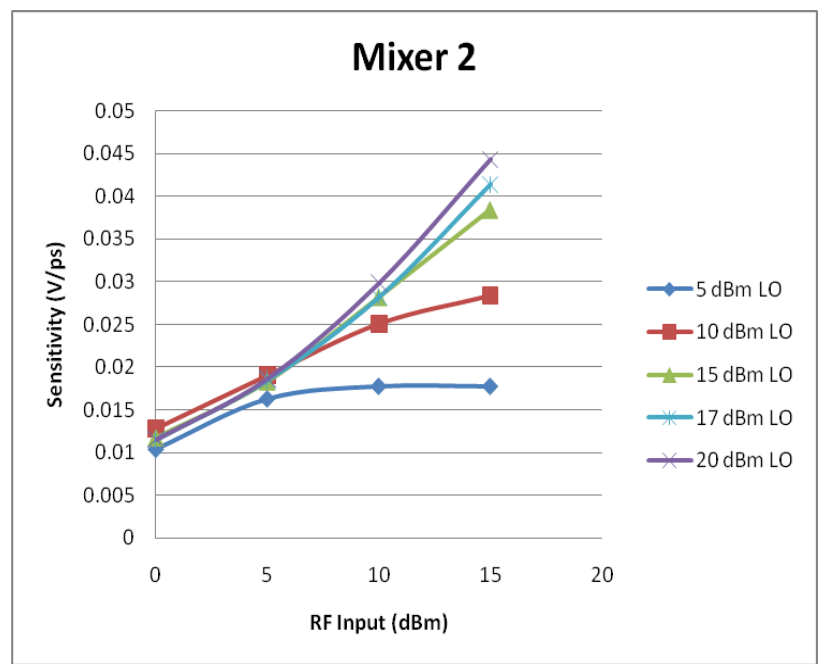

Figure 7c. Mixer 2's sensitivity vs. RF plot.

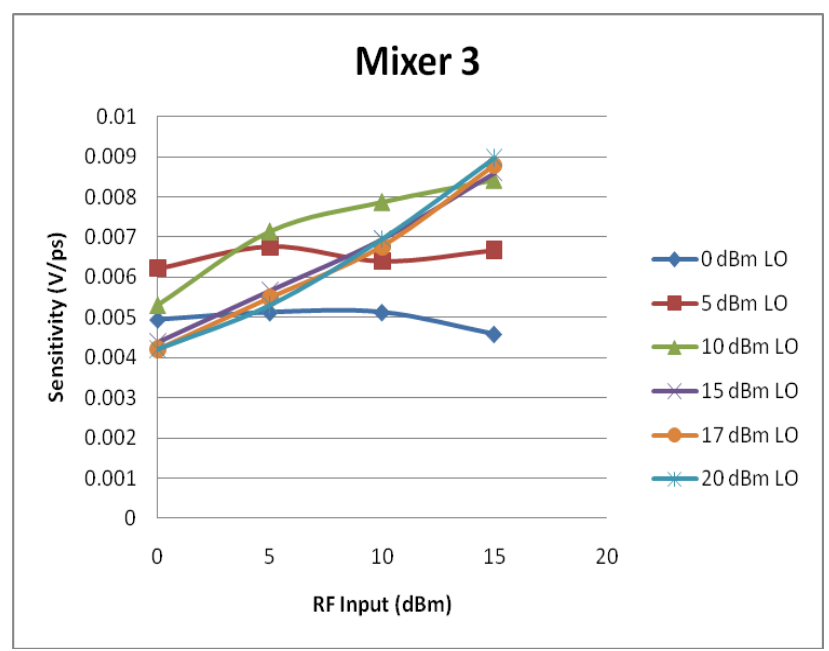

Figure 7e. Mixer 3's sensitivity vs. RF plot.

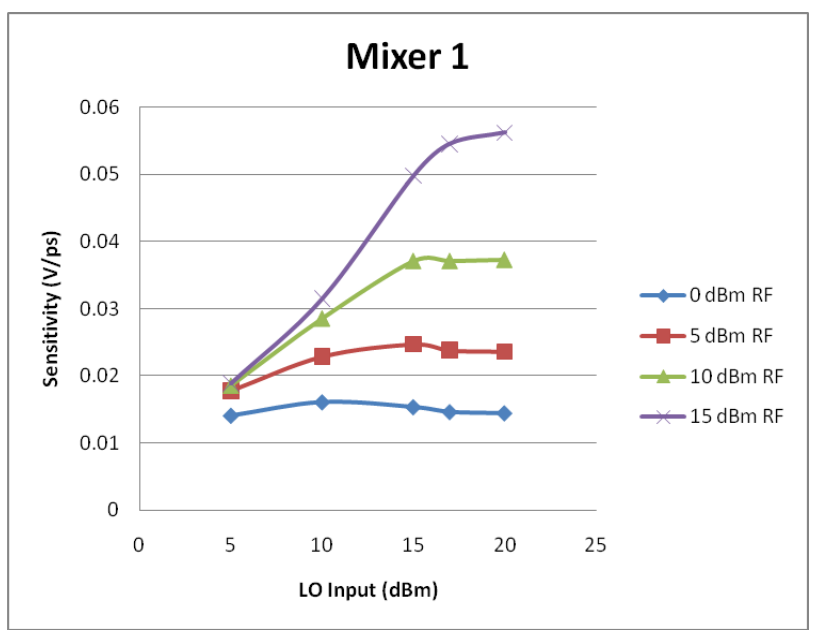

Figure 7b. Mixer 1's sensitivity vs. LO plot.

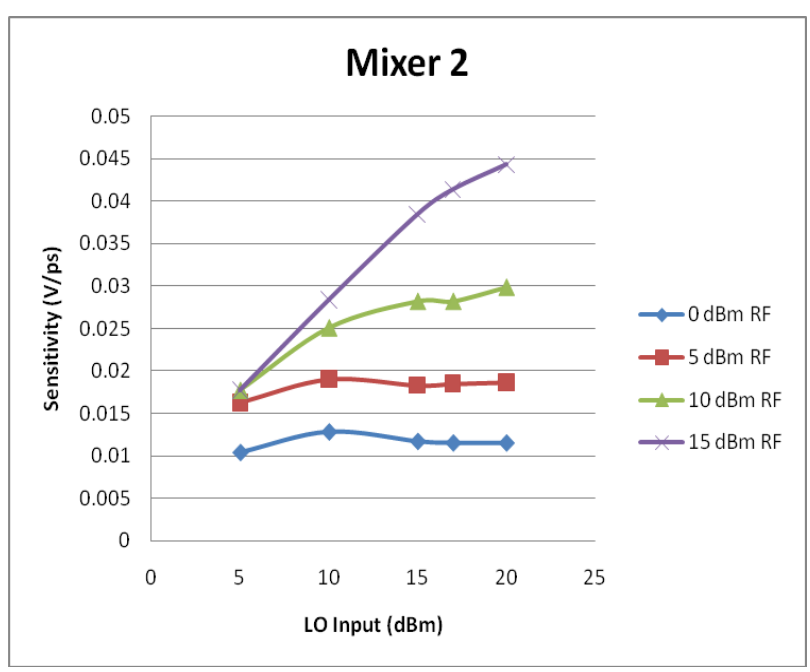

Figure 7d. Mixer 2's sensitivity vs. LO plot.

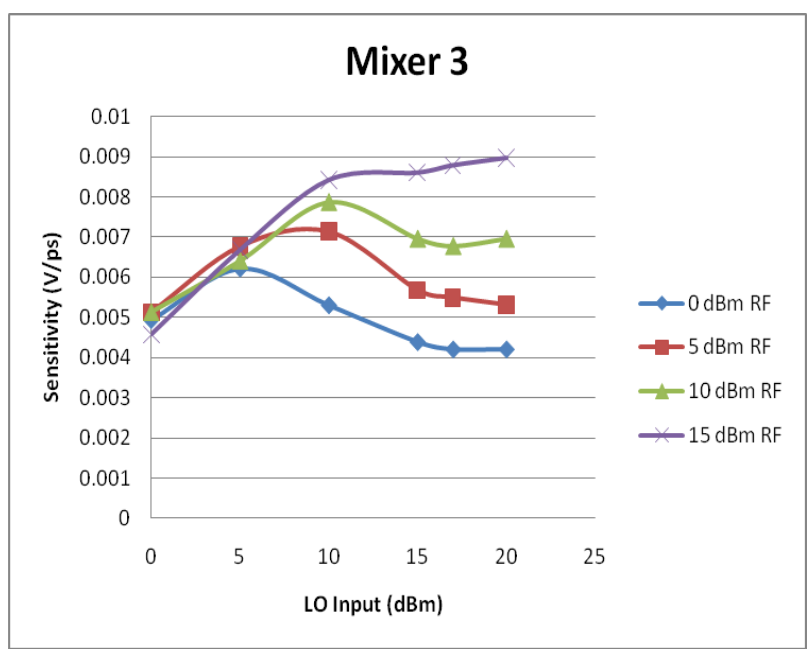

Figure 7f. Mixer 3's sensitivity vs. LO plot. 
Figures 8a-f. These graphs show the noise-to-sensitivity ratio vs. the RF input for each of the six mixer / splitter combinations.

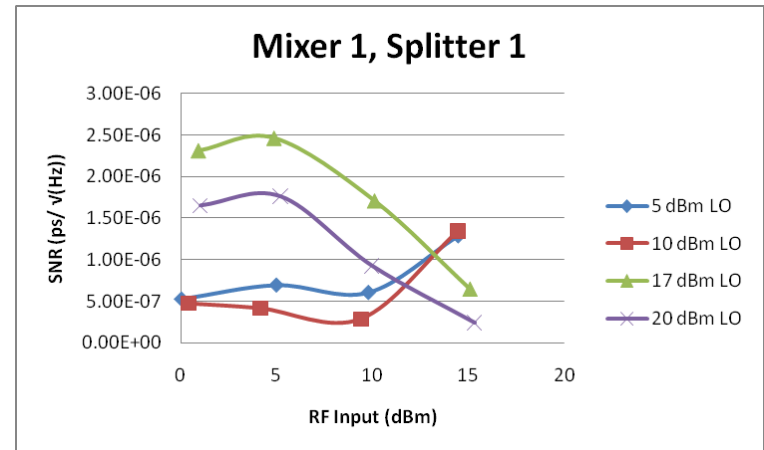

Figure 8a. Taken with a $100 \mathrm{~Hz}$ RBW

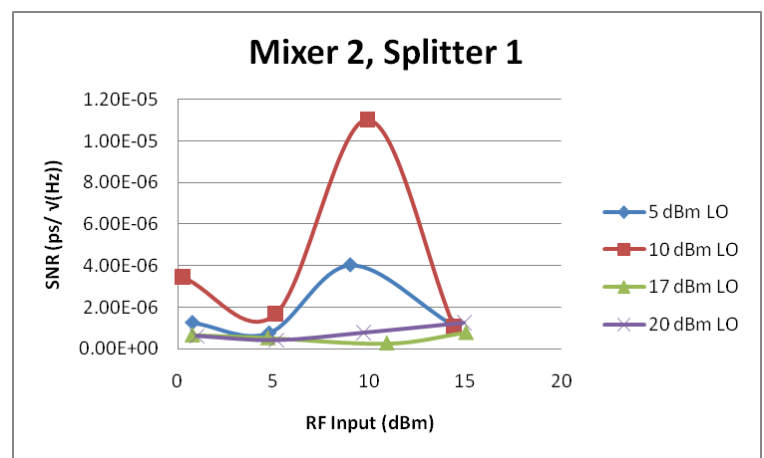

Figure 8c. Taken with a $100 \mathrm{~Hz}$ RBW

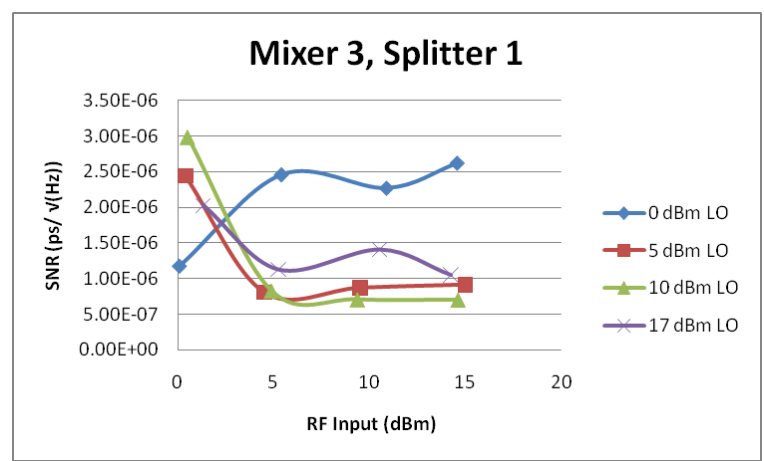

Figure 8e. Taken with a $10 \mathrm{kHz}$ RBW

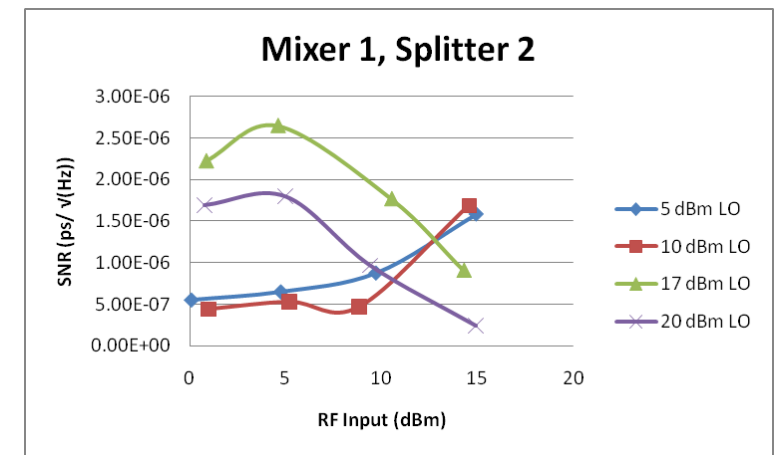

Figure 8 b. Taken with a $100 \mathrm{~Hz}$ RBW

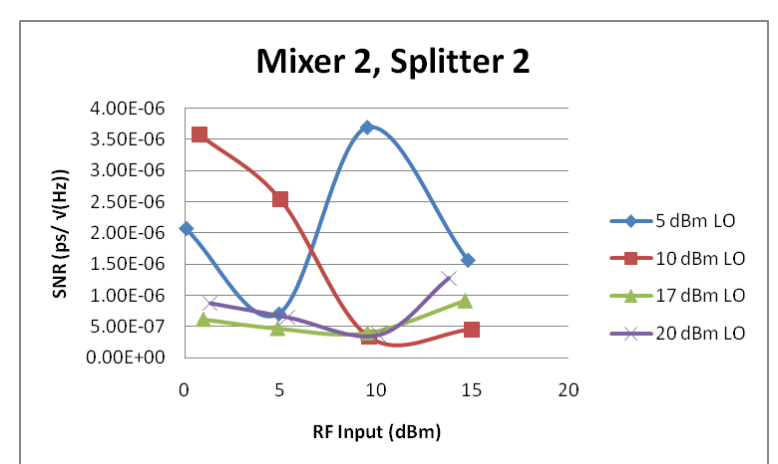

Figure $8 d$. Taken with a $100 \mathrm{~Hz}$ RBW

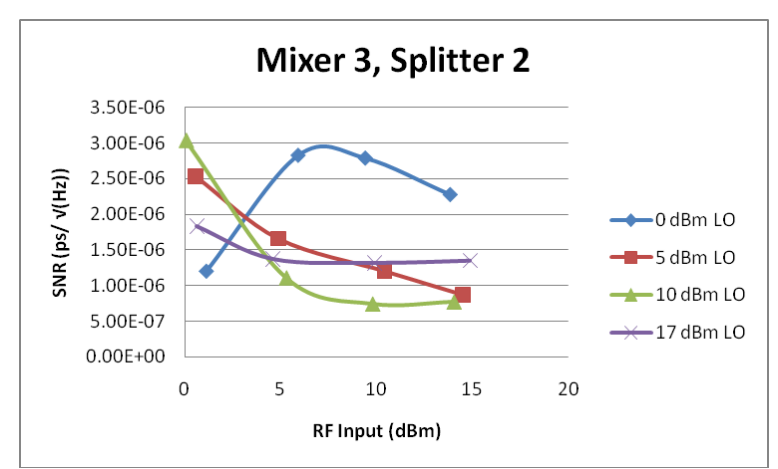

Figure 8f. Taken with a $10 \mathrm{kHz}$ RBW 